\title{
Progressive aphasia with rapidly progressive dementia in a 49 year old woman
}

\author{
John D W Greene, John R Hodges, James W Ironside, Charles P Warlow
}

Department of Clinical Neurosciences, University of Edinburgh J D W Greene C P Warlow

Neuropathology Laboratory, Department of Pathology, Western General Hospital, Edinburgh

J W Ironside

University of Cambridge Neurology Unit, Addenbrooke's Hospital and MRC Brain and Cognitive Sciences Unit, 15 Chaucer Road, Cambridge J R Hodges

Correspondence to: Dr J D W Greene, Department of Clinical Neurosciences, Western General Hospital, Crewe Road, Edinburgh EH4 2XU, Scotland, UK

Received 3 July 1998 and in revised form

3 August 1998

Accepted 10 August 1998

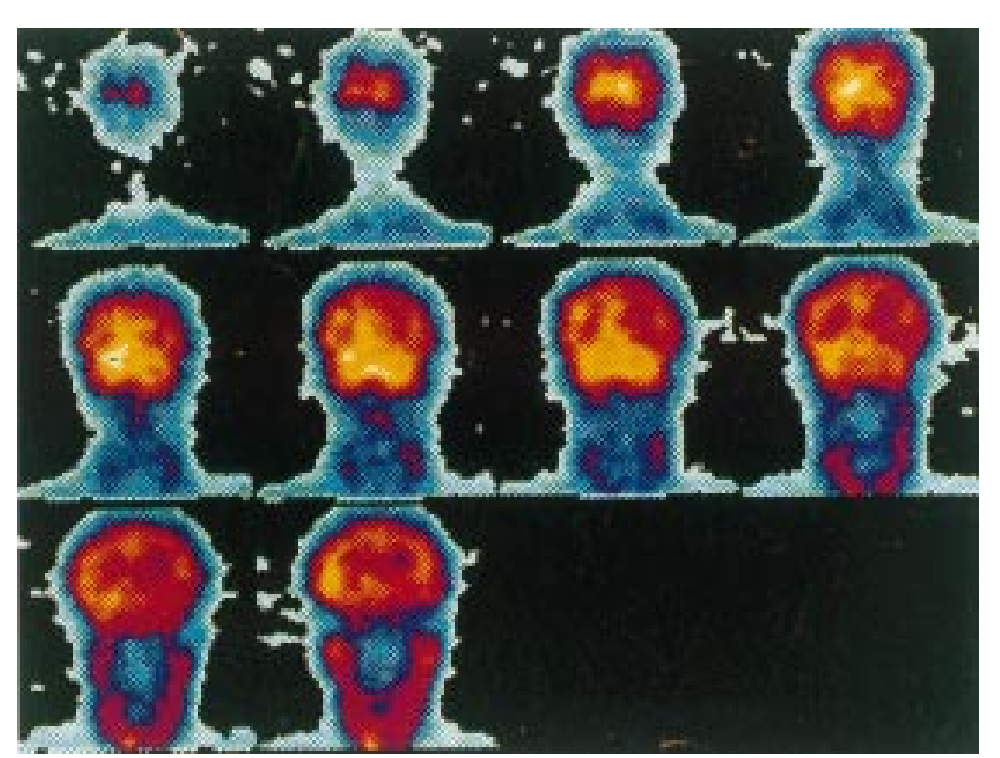

Figure 1 SPECT cerebral perfusion scan (axial cuts) showing hypoperfusion in the left

temporal and parietal and right parietal regions.

\section{Case presentation}

This 49 year old woman was referred to the Neurology Department in November 1994. Poor memory and speech difficulty had been present for about 18 months. She noticed an inability to remember the names of common household objects. In addition she complained of forgetting people's names, and noted that her speech was more hesitant, with a stammer. She could not decide what to choose from restaurant menus, she had become increasingly muddled when presented with new information or new tasks, and at times became agitated, restless, and unsettled. She had become more emotionally labile, becoming tearful at the slightest upset. She had no other symptoms. Hypertension had recently been diagnosed, and she was taking an antihypertensive drug and hormone replacement therapy for perimenopausal symptoms. There was no family history of neurological disorder. She ran her own business as a photographer. She was a non-smoker and drank about two units of alcohol a day.

On examination, there were no cardiovascular abnormalities. Blood pressure was 180/ 100. On testing higher cortical function, her speech was somewhat rambling and hesitant with word finding difficulty. Serial sevens were poor and she could not remember the name of the prime minister. In addition she had poor knowledge of current affairs and could not interpret proverbs appropriately. Cranial nerve examination was normal, there were no primitive reflexes, and the rest of the neurological examination was normal.

Initial investigations were undertaken. The following blood tests were normal: full blood count, erythrocyte sedimentation rate, $B_{12}$, folate, urea and electrolytes, glucose, liver function, thyroid function, lipids, serum immunoglobulins, vanereal disease research laboratory test, and serum copper. Brain CT and MRI were normal, as was the chest radiography. A SPECT cerebral perfusion (fig 1) disclosed markedly reduced perfusion in the left temporal and parietal and right posterior parietal regions. Electroencephalography showed underlying delta over the left hemisphere, especially over the temporal areas, with superimposed theta activity seen over the left hemisphere. Examination of CSF disclosed normal biochemistry, bacteriology, white cell count, and cytology, and there were no oligoclonal bands.

Neuropsychological assessment in April 1995 showed difficulties with all aspects of language, including comprehension, repetition, expression, naming, reading, and writing, and she had commensurate difficulties with calculation. She also showed difficulties with concentration and keeping track, memory for new material (both verbal and non-verbal), visuospatial abilities, and aspects of executive function including abstraction and planning. Her general behaviour was pleasant and appropriate, and she retained insight.

Alzheimer's disease was diagnosed. She was reviewed in the clinic in May 1995. She continued to deteriorate, and was noted to have marked expressive dysphasia. She and her family requested a second opinion. The second consultant neurologist confirmed the history. On examination he found a marked expressive and receptive dysphasia with dysgraphia. There was some evidence of apraxia. There were no focal signs and no primitive reflexes. The second neurologist also made a clinical diagnosis of Alzheimer's disease. In July 1995 she was referred to the psychiatric services. In addition to her memory and language difficulties, she complained of difficulties with her vision and 
weight loss. Her functional abilities continued to decline, and she was no longer able to shop or cook. She was reviewed in November 1995 and it was noted that her cognitive and functional decline continued relentlessly. Motor agitation and restlessness were the most prominent symptoms. She had to be admitted to a dementia assessment unit for long term care. In March 1996 she developed generalised tonic-clonic convulsions and was started on carbamazepine. She continued to deteriorate, and died on 25 March 1997.

\section{Discussion \\ Professor $\mathcal{F} R$ Hodges \\ HISTORY}

This 49 year old patient developed a fairly rapidly progressive dementia. It is a difficult age diagnostically as she is at the cusp of diseases causing dementia in young adults, and those causing dementia in later life. She had had an 18 month history of difficulty finding names, with speech hesitancy. So, are we dealing with a pure progressive aphasia, or are there signs of other cognitive deficits indicative of left or right hemisphere pathology? This is not always easy to answer. At presentation, there are hints; she did not have a pure linguistic deficit, as she also had difficulty making decisions, was easily muddled, and showed agitation, restlessness, and emotional lability. It would have been useful to know whether there were fluctuations, or any hint of a delirious component to her illness. Her history was unremarkable other than for hypertension. The lack of family history is very important; in assessing early onset dementia, this must be considered in detail. Is is not appropriate simply ask for a family history of dementia, but it is necessary to determine the age at which parents died, and ideally to obtain details on siblings, aunts, and uncles. Not infrequently a family history of a neurodegenerative disorder is unearthed. The absence of a family history does not lend support to Alzheimer's and frontotemporal dementia, although only half of early onset cases are familial.

\section{EXAMINATION}

The general examination was unremarkable and there were no focal neurological signs. On bedside cognitive testing, her speech was described as rambling and hesitant, with word finding difficulties. I would classify this as nonfluent on clinical grounds. If a patient has any degree of dysphasia, it becomes very difficult to comment on verbal memory and general intellectual ability.

INVESTIGATIONS

Routine blood tests were normal. I would routinely check thyroid autoantibodies in such a case, looking for Hashimoto's encephalopathy. Moving on to imaging, by the time the MRI was done, she was significantly cognitively impaired, with not just aphasia but evidence of more general dementia, yet the MRI was strikingly normal. I would have preferred coronal cuts, as these visualise the hippocampus and inferior temporal lobes well, and are of particular use in looking for focal lobar atrophy, but these were not provided. The SPECT cerebral perfusion was, however, severely abnormal, with striking hypoperfusion in the left hemisphere, and less marked changes in the right parietal region. Imaging is therefore narrowing down the differential diagnosis into conditions in which the brain appears grossly structurally normal, but in which the pathology is resulting in severe hypoperfusion. The CSF was normal, the lack of oligoclonal bands being important when we get to the differential diagnosis. The EEG was abnormal over the left hemisphere, but this is non-specific.

Turning to the neuropsychology, she had a global aphasia with deficits in naming, repetition, comprehension, reading and writingthat is, a non-specific pattern. Concentration was said to be poor. I would not put much weight on verbal memory, given the existing aphasia. I am really looking for hints that the cognitive impairment is more diffuse than it seems: she had impaired non-verbal memory (subserved by the right hippocampus) and impaired visuospatial skills (indicative of a parietal deficit, probably bilateral). Insight was retained.

\section{DISCUSSION}

She progressed with frightening rapidity. In the 6 months between initial clinical attendance and neuropsychology, there had been a further deterioration, and she now had a global aphasia. A diagnosis of Alzheimer's disease was made. Two months later, she was seen by a psychiatrist. The nature of her visual impairment might be critical. Was it a retinal problem (perhaps related to vasculitis), a visual agnosia (indicative of cerebral pathology), or a visual field defect? Central visual symptoms are common in Creutzfeldt-Jakob disease. Another interesting issue is her weight loss. Most rapidly progressive dementias are accompanied by weight loss. However, systemic features are also common in Creutzfeldt-Jakob disease. We also have to consider systemic diseases, notably neoplastic and endocrine disorders.

Going back to the timing of her illness, the prodrome was 18 months. A year after being first seen in the clinic, we are told that she was no longer able to cook or shop, and was markedly agitated and restless. Within 12 months, she had gone from being able to self report at the clinic that she could no longer order food in a restaurant to requiring long term care. Sixteen months after presentation, she is said to have had seizures. Any cortical form of dementia can cause seizures. She died 28 months after presentation.

\section{Summary of presentation}

The crucial features are these. She was only 49. There was no family history. She developed a progressive dysphasic syndrome 18 months before presentation. When seen, she exhibited emotional lability and agitation. On imaging, there was a dissociation between the normal MRI and the very abnormal SPECT. She developed global cognitive impairment within 6 months and showed a rapid downhill course 
with seizures, and died two and a half years after her first symptoms. Before going on to the differential diagnosis, I would like to say a few words about progressive aphasia.

PROGRESSIVE APHASIA

This was first described by Pick in the late 19th century. Most people associate his name with frontal dementia, but he was principally interested in language and the focal manifestations of degenerative disease. His patients with progressive aphasia seemed to have lost the meaning of words, and exhibited striking left temporal lobe atrophy. His work had been largely forgotten, and Pick's disease has until recently become synonymous, wrongly, with frontal dementia. In 1982, Mesulam resurrected the concept of slowly progressive aphasia without dementia. ${ }^{1}$ These patients had been aphasic for 6-10 years, yet were still self caring. Since then, over 100 cases have been reported. The diagnostic criteria are as follows: progressive speech decline for more than 2 years yet with normal activities of daily living, and neuropsychological evidence of a pure aphasic syndrome. There are two subgroups. One is progressive non-fluent aphasia. Speech is broken up, with phonological paraphasias and disorders of syntax. It tends to be slowly progressive. The other form is progressive fluent aphasia, termed semantic dementia. In this disorder, the phonological and syntactic structure of language remains normal but patients are profoundly anomic and show many semantic errors. This can progress over several years. ${ }^{23}$

The pathology of progressive aphasia is varied. Mesulam found non-Alzheimer's, nonPick pathology in his series. There is often spongiosis, but usually no specific markers of Alzheimer's or Pick pathology. As more cases have been reported, the histology has been varied. In Alzheimer's disease, there is initially loss of episodic memory, with language disorders occurring later. There are, however, four cases of slowly progressive aphasia where histology has confirmed Alzheimer's disease. This may be fluent ${ }^{45}$ or non-fluent. ${ }^{67}$ When Alzheimer's disease presents with fluent aphasia, there are usually clear structural changes on MRI. Also, in the present case, the progression is much more rapid than is typical for Alzheimer's disease. Progressive aphasia may also be due to Pick's disease..$^{8-11}$ There have also been two case reports in which progressive aphasia was due to Creutzfeldt-Jakob disease. ${ }^{12}{ }^{13}$

CAUSES OF A RAPIDLY PROGRESSIVE DEMENTIA What diseases can present with aphasia and yet cause a rapidly progressive dementia? Creutzfeldt-Jakob disease is a distinct possibility. To mention first sporadic, or old variant Creutzfeldt-Jakob disease, Brown et $a l^{14}$ noted that the average age of onset is 60 , the younger patients being in their late 40s. The mean duration of the illness is 4 to 6 months, although a small proportion live longer. Brown et al also reported on so-called longer duration Creutzfeldt-Jakob disease. ${ }^{15}$ These patients tended to be younger, to have less typical
EEGs, to have less in the way of myoclonus, and to have less transmissibility than typical Creutzfeldt-Jakob disease. Histology showed spongiform changes, not dissimilar to that described as spongiform changes in focal aphasia (see above). Central disturbances of vision are also common in Creutzfeldt-Jakob disease, due to occipital cortical involvement, which does not commonly occur in Alzheimer's disease. If a patient is severely demented, and the MRI is normal, this always makes me think of Creutzfeldt-Jakob disease. I have less experience of SPECT in this disease. It is apparently very abnormal in new variant Creutzfeldt Jakob disease ${ }^{16}$ and in old variant disease could cause the MRI-SPECT discrepancy in this patient. Moving on to new variant Creutzfeldt Jakob disease, so far all the cases have been younger than 45 , and many have been in their 20s. They tend to present with psychiatric $^{17}$ or sensory symptoms. They also exhibit ataxia, with involuntary movements ${ }^{18}$ and some have abnormal MRIs.

There are clearly other disorders which cause rapidly progressive dementia, but I am dismissing many on the basis of the normal MRI and CSF and the lack of encephalopathic features. There are peculiar chronic encephalitic illnesses, such as Rasmussen's encephalitis and Landau-Kleffner syndrome, which are predominantly diseases of childhood and adolescence, although they can present in later life. Cerebral vasculitis can be a great mimic, is a great favourite in clinicopathological conferences, and can present focally. In this case, however, there are no stroke or multiple sclerosis-like episodes, and the MRI was normal with no oligoclonal bands on CSF, so I think that it is almost inconceivable.

I also considered Hashimoto's encephalopathy. It tends to affect young to middle aged women. It usually begins abruptly, and typically causes an encephalopathic illness. There can be focal signs, and headaches are common. The CSF is usually abnormal. Thyroid function can be normal, so it is imperative to check for thyroid autoantibodies. The progressive unremitting course in this patient is very much against this as a diagnosis.

Whipple's disease is a possibility, especially given the history of weight loss. These patients also tend to be encephalopathic. Movement disorders, including oculomasticatory myorhythmia, and brain stem symptoms, are described in this condition.

In a rapidly progressive dementia, neoplastic disorders must also be considered. One can be caught out in a patient with no focal neurology and an initially normal MRI; in a few months time, repeat imaging may show an infiltrative multifocal glioma. I do not think that this patient could have developed focal signs without being brought to medical attention. Paraneoplastic limbic encephalitis tends to present acutely with a profound and pure amnesic syndrome. Examination of CSF tends to give normal results and changes in MRI have been reported. There are also reports of more generalised dementias and more encephalitic 
illnesses being paraneoplastic syndromes, but these are rather ill defined.

Alzheimer's disease can occasionally cause a rapidly progressive dementia. In Oxford in the early days of Creutzfeldt-Jakob disease surveillance, Alzheimer's disease was the second commonest diagnosis after Creutzfeldt-Jakob disease. It can cause myoclonus and seizures, and can present focally. In presenile cases, one in three have a positive family history. The younger the onset, the more likely they are to have a positive family history. It can present with aphasia, which is a poor prognostic sign. I would expect an abnormal MRI and the course in the present case is beyond our experience of several hundred cases.

Cortical Lewy body disease can be mistaken for Creutzfeldt-Jakob disease due to its rapid progression. However, patients tend to be older, they are parkinsonian, and show fluctuations. It is an unlikely diagnosis here.

\section{Summary}

I think that this is a patient with an unusual presentation of Creutzfeldt-Jakob disease. It is probably sporadic or "old variant".

Questions

"Could you go into more detail about your views of the normal MRI and abnormal SPECT?"

This structure-function disparity is seen in encephalopathic illnesses, in which a disturbance of brain metabolism down regulates blood flow. In this patient, due to the course of the illness with lack of fluctuations and other features of delirium, I have ruled this out. The MRI-SPECT dissociation is also compatible with Creutzfeldt-Jakob disease where there is a severe disruption of cerebral architecture, not enough to cause atrophy, but sufficient to disrupt metabolism and blood flow.
"Would you have done a biopsy?"

If I had thought of Creutzfeldt-Jakob disease, I would have been keen on a biopsy. As you know, this is a contentious and difficult area. We have biopsied four patients with atypical dementias in seven years, typically in the young. It has, on the whole, been unrewarding. It did not alter management much, but at least we were reassured that we were not missing something treatable.

"Your main differential was an uncommon presentation of an uncommon disease (Creutzfeldt-Jakob disease) or an uncommon presentation of a common disease (Alzheimer's disease). Why did you pick the former?"

Maybe I'm too much in Creutzfeldt-Jakob disease mode rather than clinical mode. There were, however, other features that put me off Alzheimer's disease. When she was cognitively very impaired, the MRI was strikingly normal (although there are reservations regarding the lack of coronal cuts). If Alzheimer's disease is the cause of global cognitive impairment, then I would have expected to see more abnormalities on MRI. Also, in our Cambridge Alzheimer's disease cohort, none have died within 2 years of diagnosis. The rapidity of progression is, therefore, out of the ordinary for Alzheimer's disease.

If you look at Brown's Creutzfeldt-Jakob disease series in the mid-1980s, the prognosis is bimodal; there is a big mortality peak at 4-6 months, but then a long tail, with some patients showing a protracted course. Admittedly, some of these were familial, but not all. They report a long prodromic phase, followed by a rapid downhill phase. This is the sort of course she followed. There are also well documented cases of Creutzfeldt-Jakob disease presenting with progressive aphasia.

"What are your opinions regarding the EEG?"

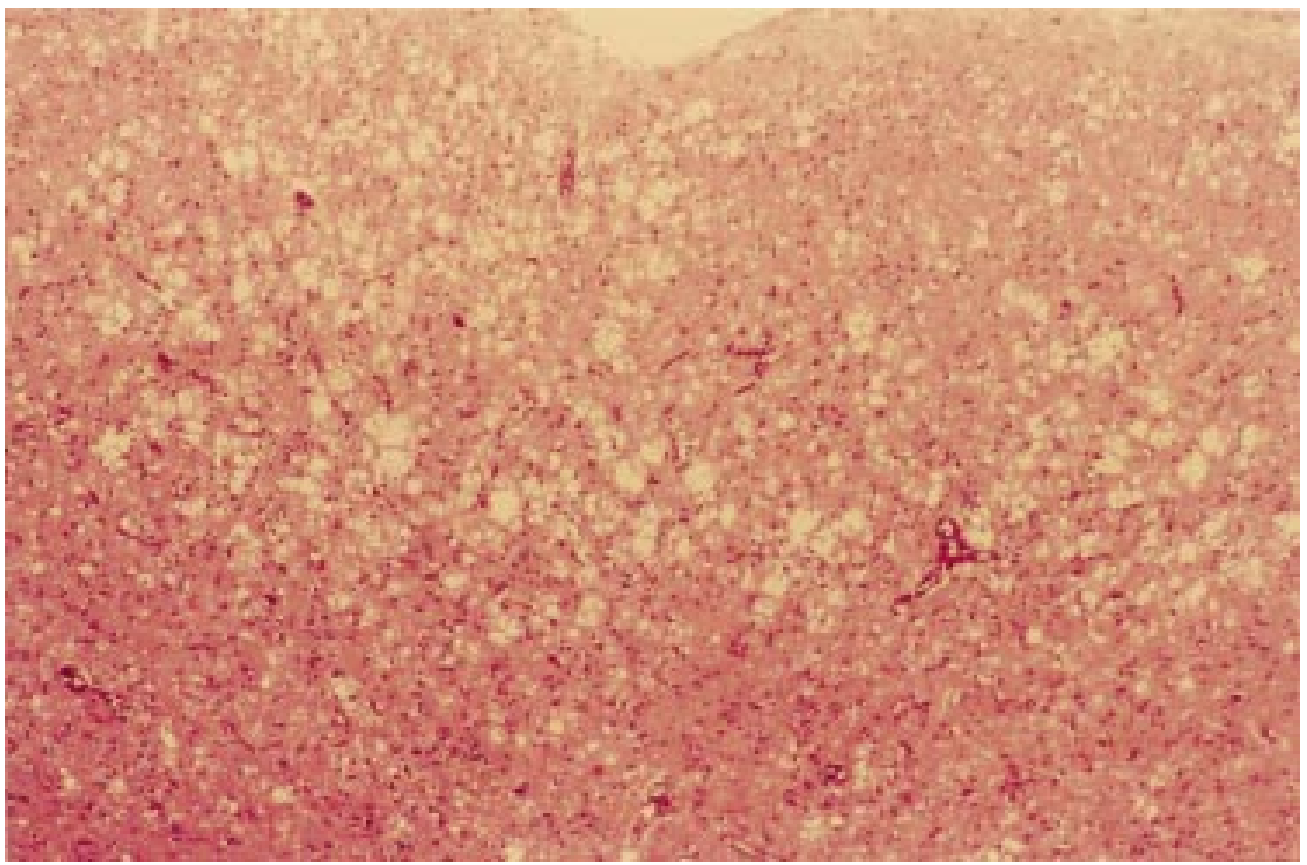

Figure 2 Widespread spongiform change is present in the frontal cortex, with confluent vacuolation (haematoxylin and eosin; originally $\times 200$ ). 


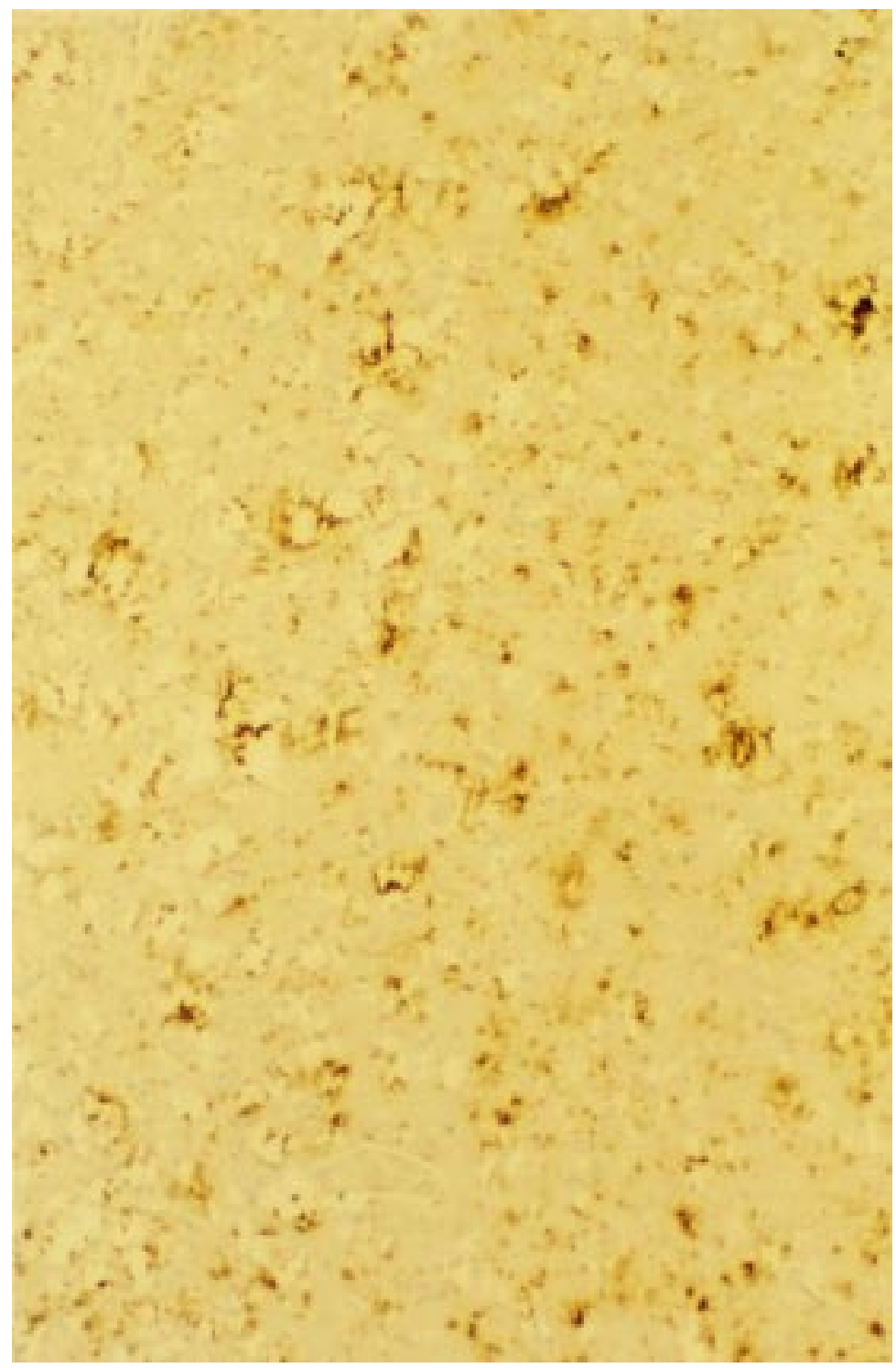

Figure 3 Immunocytochemistry for prion protein shows positive staining (brown) in a widespread distribution in the frontal cortex, with focal dense aggregates around areas of confluent spongiform change (KG9 monoclonal antibody; originally $\times 200)$.

It is more focally abnormal than the diffuse slowing we often see in Alzheimer's disease. My experience of EEGs in dementia is not great but I think that in this case, the EEG is rather abnormal even for focal Alzheimer's disease.

\section{Dr $\mathcal{F} W$ Ironside}

At postmortem examination, there was evidence of muscle wasting and bronchopneumonia but no other abnormalities were noted in the thorax or abdomen. The brain weighed $1054 \mathrm{~g}$ and was markedly atrophic. Coronal sections confirmed significant cortical atrophy in a widespread distribution, with accompanying ventricular dilatation. The hippocampi were atrophied symmetrically, but only to a mild degree. No evidence of lobar atrophy was noted and there was no sign of tumour, infection, haemorrhage or infarction. The meningeal vessels were unremarkable. No selective atrophy of the lentiform nuclei or thalamus was noted. The cerebellum appeared unremarkable on cross section, as did the brain stem.
Histology of the frontal and temporal cortex in progressive aphasia often shows marked neuronal loss, but this was not prominent here. There was evidence of spongiosis, with small areas of vacuolation in the neuropil. In terms of differential diagnosis there was no evidence of amyloid plaques or neurofibrillary tangles to suggest a diagnosis of Alzheimer's disease and no Lewy bodies, Pick bodies or other inclusions were identified on immunocytochemistry for ubiquitin or tau.

The hippocampus showed widespread vacuolation and histological examination of the temporal and inferior frontal cortex showed areas of confluent vacuolation, with occasional neuronal vacuolation compatible with a diagnosis of spongiform encephalopathy. None of the "florid" plaques characteristic of new variant Creutzfeldt-Jakob disease were present. With regard to the topography of the vacuolation, it was most severe in the frontal and temporal cortex, with severe changes in the inferior temporal gyri (fig 2). This was accompanied by marked neuronal loss and astrocytosis. However, spongiform change was present in a widespread distribution including the occipital cortex, caudate nucleus and thalamus in a patchy distribution. The cerebellum showed focal spongiform change in the molecular layer accompanied by a loss of granular neurons, with patchy gliosis and preservation of Purkinje cells. No amyloid plaques were identified in the cerebellum. The blood vessels throughout the brain were unremarkable and showed no sign of amyloid angiopathy. Thus the diagnosis on routine sections is spongiform encephalopathy (Creutzfeldt-Jakob disease).

To investigate conditions causing focal spongiosis in the brain, immunocytochemistry for prion protein was performed, with widespread positivity in a perivacuolar distribution, particularly in the frontal and temporal cortex (fig 3).

None of the characteristic plaques of new version Creutzfeldt-Jakob disease were present in either the cerebrum or cerebellum. Analysis of the prion protein gene in this patient showed no pathogenic mutations, with homozygosity for methionine at codon 129. There was no history of iatrogenic exposure to the Creutzfeldt-Jakob disease agent. In summary, the diagnosis is sporadic Creutzfeldt-Jakob disease.

\section{QUESTIONS}

"Is it unusual for the brain to be so atrophic in Creutzfeldt-Jakob disease? Our pathologist says the brain does not atrophy in CreutzfeldtJakob disease".

$\operatorname{Dr} \mathcal{F} W$ Ironside - This is true in most rapidly progressive cases of Creutzfeldt-Jakob disease. In unusual cases such as this with a longer history, cerebral atrophy is often more pronounced. In Japan, where the average survival of patients with Creutzfeldt-Jakob disease is much longer than in the United Kingdom, cerebral atrophy is common, with brain weights not infrequently reduced to 800-900 g.

Dr Knight (National CFD Surveillance Unit, Edinburgh) - We rarely see atrophy on MRI in 
Creutzfeldt-Jakob disease. Another point is that we find clearly genetic cases with the appropriate prion protein mutation despite there being no family history. I think that there are three types of patients with long progression Creutzfeldt-Jakob disease. In one group, they simply have a long, slow deterioration. In the second group, the initial symptoms are paroxysmal, and may mimic transient ischaemic attacks, but then develop a rapidly progressive course. In the third group, there is a short very rapid decline, but they then survive in an almost persistent vegetative state. I would also say that there are not much data regarding SPECT imaging in Creutzfeldt-Jakob disease.

\section{Conclusions}

Professor $\mathcal{F} R$ Hodges' diagnosis:

Sporadic Creutzfeldt-Jakob disease

Pathological diagnosis

Creutzfeldt-Jakob disease

\section{Comment}

Primary progressive aphasia may sometimes remain an isolated phenomenon, without accompanying non-linguistic cognitive deficits. ${ }^{1319}$ The issue of whether all patients with progressive aphasia eventually develop more generalised dementia remains controversial, but there is little doubt that, in many instances, progressive language disorder is the harbinger of dementia. Moreover, this presentation is not specific for any one disease ${ }^{20}$; patients with Pick disease, ${ }^{8-11}$ Alzheimer's disease, ${ }^{4-7}$ and Creutzfeldt-Jakob disease ${ }^{121321}$ have been described with progressive aphasia.

Although Creutzfeldt-Jakob disease may present with focal neurological signs or symptoms, neuropathological studies tend to show diffusely distributed disease. ${ }^{22}$ Matthews ${ }^{23}$ states that the "commonest clearly focal abnormality of higher cortical dysfunction" in Creutzfeldt-Jakob disease is aphasia, often of sudden onset. Kirshner et al presented postmortem data on three patients with relatively isolated progressive aphasia. ${ }^{24}{ }^{25}$ Interestingly, two had left perisylvian foci of status spongiosis restricted to cortical layer two. In retrospect, these cases may well have been CreutzfeldtJakob disease. It is perhaps unsurprising that patients with early disease and focal symptoms and signs might show focal rather than diffuse pathology. Mandell et $a l^{13}$ describe a patient with Creutzfeldt-Jakob disease who gradually developed progressive fluent aphasia as the major manifestation for almost a year. Another patient with sudden onset of non-fluent aphasia due to Creutzfeldt-Jakob disease was described by Kirk and Ang. ${ }^{21}$ As in our patient, it is interesting that their patient not only was non-fluent, but had a normal CT and MRI yet abnormal SPECT.

In summary, we have shown that in patients with progressive non-fluent aphasia which generalises to dementia, the list of possible histological diagnoses includes CreutzfeldtJakob disease. In such cases, neuropathological studies are essential for diagnosis and should include immunocytochemistry for prion protein.

1 Mesulam MM. Slowly progressive aphasia without generalised dementia. Ann Neurol 1982;11:592-8.

2 Hodges JR, Patterson K, Oxbury S, et al. Semantic dementia: progressive fluent aphasia with temporal lobe atrophy. Brain 1992;115:1783-806.

3 Hodges JR, Patterson K. Nonfluent progressive aphasia and semantic dementia: A comparative neuropsychological study. Fournal of the International Neuropsychological Society 1996;2:511-24.

4 Kempler D, Metter EJ, Riege WH, et al. Slowly progressive aphasia. F Neurol Neurosurg Psychiatry 1990;53:987-93.

5 Pogacar S, Williams RS. Alzheimer's disease presenting as slowly progressive aphasia. Rhode Island Med $\mathcal{F}$ 1984;67: $181-5$.

6 Greene JDW, Patterson K, Xuereb J, et al. Alzheimer disease and nonfluent progressive aphasia. Arch Neurol 1996;53 $1072-8$

7 Green J, Morris JC, Sandson J, et al. Progressive aphasia: a precursor of global dementia? Neurology 1990;40:423-9.

8 Wechsler AF, Verity MA, Rosenscheim S, et al. Pick's disease. Arch Neurol 1982;39:3287-90.

9 Holland AL, McBurney DH, Moossy J, et al. The dissolution of language in Pick's disease with neurofibri

0 Caplan LR, Richardson EPJ. Case records of the Massachusetts General Hospital: weekly clinicopathological exersetts General Hospital: weekly clinicopat
cises. $N$ Engl f Med 1986;314:1101-11.

11 Graff-Radford NR, Damasio AR, Hyman BT, et al. Progressive aphasia in a patient with Pick's disease. Neurology 1990;40:620-6

12 Shuttleworth EC, Yates AJ, Paltan-Ortiz JD. CreutzfeldtJakob disease presenting as progressive aphasia. F Natl Med Assoc 1985;77:649-55.

13 Mandell AM, Alexander MP, Carpenter S. CreutzfeldtJakob disease presenting as isolated aphasia. Neurology 1989;39:55-8.

14 Brown P, Cathala F, Castaigne P, et al. Creutzfeldt-Jakob disease: clinical analysis of a consecutive series of 230 neuropathologically verified cases. Ann Neurol 1986;20:597602 .

15 Brown P, Rodgers-Johnson P, Cathala F, et al. Creutzfeldt Jakob disease of long duration. Clinicopathological characteristics, transmissibility and differential diagnosis. Ann teristics, transmissibility

16 de Silva R, Patterson J, Hadley D, et al. Single photon emission computed tomography in the identification of new variant Creutzfeldt-Jakob disease: case reports. BMF 1998; 316:593-4.

17 Zeidler M, Johnstone EC, Bamber RW, et al. New variant Creutzfeldt-Jakob disease: psychiatric features. Lancet 1997;350:908-10.

18 Zeidler M, Stewart GE, Barraclough CR, et al. New variant Creutzfeldt-Jakob disease: neurological features and psychiatric tests. Lancet 1997;350:903-7.

19 Mesulam MA. Primary progressive aphasia: differentiation from Alzheimer's disease. Ann Neurol 1987;37:1448-553.

20 Mendez MF, Zander BA. Dementia presenting with aphasia. $\mathcal{F}$ Neurol Neurosurg Psychiatry 1991;54:542-5.

21 Kirk A, Ang LC. Unilateral Creutzfeldt-Jakob disease presenting as rapidly progressive aphasia. Can f Neurol Sci 994;21:350-2.

22 Kirschbaum WR. Fakob-Creutzfeldt disease. New York: American Elsevier, 1968

23 Matthews WB. Creutzfeldt-Jakob disease. In: Vinken PJ, Bruyn GW, Klawans HL, et al, eds. Neurobehavioural disorders. Handbook of clinical neurology. Amsterdam: Elsevier Science, 1985;46:289-99.

24 Kirshner HS, Webb WG, Kelly MP, et al. Language disturbance: an initial symptom of cortical degenerations and dementia. Ann Neurol 1984;41:491-6.

25 Kirshner HS, Tanridag O, Thurman L, et al. Progressive aphasia without generalised dementia: two cases with focal spongiform degeneration. Ann Neurol 1987;22:527-32. 